\title{
Local neuroinflammation and the progression of Alzheimer's disease
}

\author{
Patrick L McGeer and Edith G McGeer \\ Kinsmen Laboratory of Neurological Research, Department of Psychiatry, University of British Columbia, \\ Vancouver, British Columbia, Canada
}

\begin{abstract}
Postmortem immunohistochemical studies have revealed a state of chronic inflammation limited to lesioned areas of brain in Alzheimer's disease. Some key actors in this inflammation are activated microglia (brain macrophages), proteins of the classical complement cascade, the pentraxins, cytokines, and chemokines. The inflammation does not involve the adaptive immune system or peripheral organs, but is rather due to the phylogenetically much older innate immune system, which appears to operate in most tissues of the body. Chronic inflammation can damage host tissue and the brain may be particularly vulnerable because of the postmitotic nature of neurons. Many of the inflammatory mediators have been shown to be locally produced and selectively elevated in affected regions of Alzheimer's brain. Moreover, studies of tissue in such degenerative processes as atherosclerosis and infarcted heart suggest a similar local innate immune reaction may be important in such conditions. Much epidemiological and limited clinical evidence suggests that nonsteroidal anti-inflammatory drugs may impede the onset and slow the progression of Alzheimer's disease. But these drugs strike at the periphery of the inflammatory reaction. Much better results might be obtained if drugs were found that could inhibit the activation of microglia or the complement system in brain, and combinations of drugs aimed at different inflammatory targets might be much more effective than single agents. Journal of NeuroVirology (2002) 8, 529-538.
\end{abstract}

Keywords: chemokines; complement; cytokines; microglia; NSAIDs; pentraxins

\section{Introduction}

Classically, neuroinflammation has been equated with the types of cellular reactions observed in such chronic infections as tabes dorsalis, tuberculosis of the central nervous system (CNS), or polio virus (Haymaker and Adams, 1982). The CNS is invaded by leukocytes, especially T cells, and monocytes. Studies on Alzheimer's disease (AD) have led to a major reevaluation of this classical concept of what consti-

Address correspondence to Dr. Patrick L McGeer, Kinsmen Laboratory of Neurological Research, University of British Columbia, 2255 Wesbrook Mall, Vancouver, BC, V6T 1Z3, Canada. E-mail: mcgeerpl@interchange.ubc.ca

The authors' research has been supported by grants from the Jack Brown and Family A.D. Research Fund and the Alzheimer Society of Canada, as well as donations from The Friends of UBC and individual British Columbians.

Received 22 February 2002; accepted 23 April 2002. tutes neuroinflammation. The lesions of AD are sterile, and there is little evidence of an adaptive immune response. Yet the lesions are characterized by the expression of a wide variety of molecules that are established through traditional immunological studies as inflammatory mediators. These molecules are locally generated and are based on innate host defenses. Such CNS defenses are, of course, also expressed in response to a viral attack, but these have been overlooked due to the prominent peripheral immune reaction.

Whether local or systemic, the host response may be overly aggressive, resulting in damage to host tissue. The classical concept of such misdirection is that it must be due to an autoimmune response, where antibodies or rogue T-cell lines are cloned against host proteins. But there is a much broader phenomenon, not recognized in classical immunity, where localized, innate immune responses damage viable host tissue. We define this as autotoxicity (McGeer and 
McGeer, 2000). It is this autotoxic phenomenon that comes into play in AD. Evidence is accumulating that autotoxicity also plays a significant role in such chronic degenerative conditions as atherosclerosis and osteoarthritis, as well as in other chronic neurodegenerative diseases such as amyotrophic lateral sclerosis (ALS) and Parkinson's disease (McGeer et al, 2001).

In this review, we will discuss broad categories of molecules associated with inflammation and how they are marshalled in AD. It will become apparent that a remarkable number of inflammatory mediators, once considered to be in the exclusive domain of circulating leukocytes and peripheral immune organs, are locally produced in brain, many of them even by neurons. The fact that brain, often described as an immunologically privileged organ because of isolation by the blood-brain barrier, expresses a broad spectrum of inflammatory mediators has led to a wider examination of peripheral, nonimmune tissues, where similar expressions have been identified. The information generated by examination of $\mathrm{AD}$ brain tissue is thus leading to broader concepts of immunology, and specific insights into new targets for therapy.

\section{Complement}

$\mathrm{AD}$ is the first disease where vigorous activation of complement in the absence of antibodies was shown to occur, with a resulting damage to host tissue. It is therefore the prime example of an autotoxic disorder. A key finding regarding the mechanism of complement activation was that of Rogers et al (1992), who demonstrated that amyloid protein $(\mathrm{A} \beta)$, when aggregated, was a strong complement activator. Thus, the senile plaques of $\mathrm{AD}$ have a unique activator of complement. In addition, the complement cascade can be activated by the pentraxins, amyloid P (AP), and C-reactive protein (CRP), which are up-regulated in affected regions of AD brain (Yasojima et al, 2000), as well as in atheroscleotic plaques (Yasojima et al, 2001).

Complement is a sophisticated attack system designed to destroy invaders and to assist in the phagocytosis of waste materials. The adaptive immune system makes great use of complement through antibodies that orchestrate the humoral response. But the origins of complement trace back as far as sponges, predating the development of the adaptive immune system, which is an invention of vertebrates (Lambris, 1993).

The complement system has components to carry out four major functions: recognition, opsonization, inflammatory stimulation through anaphylatoxins, and direct killing through the membrane attack complex. The classical pathway is activated by attachment of C1q to a target, causing C1 dissociation. Opsonization then takes place, first by amplification through a cascade of proteases (C1r, C1s, C4, C2, C3) and then by attachment of the cleavage products C4b and C3b to exposed sites close to the C1q binding site. Covalent bonds are formed between thiol groups exposed on these cleavage fragments and hydroxyl or amino groups on the target. The attached fragments then become ligands for complement receptors on phagocytes. In the case of brain, these phagocytes are microglia. If the complement system is fully activated, it proceeds to assemble the terminal components (C5b, C6, C7, C8, C9) into the lytic macromolecule C5b-9, known as the membrane attack complex. This complex inserts into the limiting membrane of viable cells, potentially leading to lysis and cell death. Meanwhile, the small fragments C3a, C4a, and C5a, known as anaphylotoxins, stimulate inflammation. So the overall cascade identifies, opsonizes, and destroys its target, while dispatching messengers to seek help.

The tangles and plaques of $\mathrm{AD}$ are clearly marked with the complement fragments C4d and C3d (Ishii and Haga, 1984; Eikelenboom et al, 1989; McGeer et al, 1989). Little or no such staining is seen in control brain. Dystrophic neurites in AD brain are immunostained for the membrane attack complex (Itagaki et al, 1994; Webster et al, 1997b), indicating autolytic attack. Again such staining is not seen in control brains. There are protective mechanisms that defend host cells against spurious activation of complement and against self damage when complement is activated. These include C1 inhibitor, C4-binding protein, decay-accelerating factor, membrane cofactor protein (CD46), and protectin (CD59). However, although the mRNAs for complement proteins are sharply upregulated in affected regions of AD brain (Figure 1) (Yasojima et al, 1999b), those for C1 inhibitor and CD59 are not (Yasojima et al, 1999a). Thus, there is no compensatory inhibitory up-regulation to protect host brain tissue in $\mathrm{AD}$, and it appears as if neurites are being progressively destroyed by complement self-attack.

Up-regulation of the mRNAs for the complement proteins and immunohistochemical evidence of their presence have also been found in atherosclerotic plaques (Yasojima et al, 2001) and infarcted heart (Yasojima et al, 1998). In atherosclerotic plaques, there was sharp up-regulation of complement protein mRNAs but not the defensive proteins, C1 inhibitor, decay accelerating factor, CD46, C4-binding protein, or CD59 (Yasojima et al, 2001). As with AD brain, complement is activated in atherosclerotic plaques with evidence of autodestruction and an absence of host defense.

Taken together, these data indicate that complement activation exacerbates the pathology in such common conditions as $\mathrm{AD}$ and atherosclerosis, suggesting that complement inhibitors might be effective anti-inflammatory agents. There are many steps in the complement cascade so that multiple opportunities exist for therapeutic intervention. The intervention, however, should not be of a nature that 


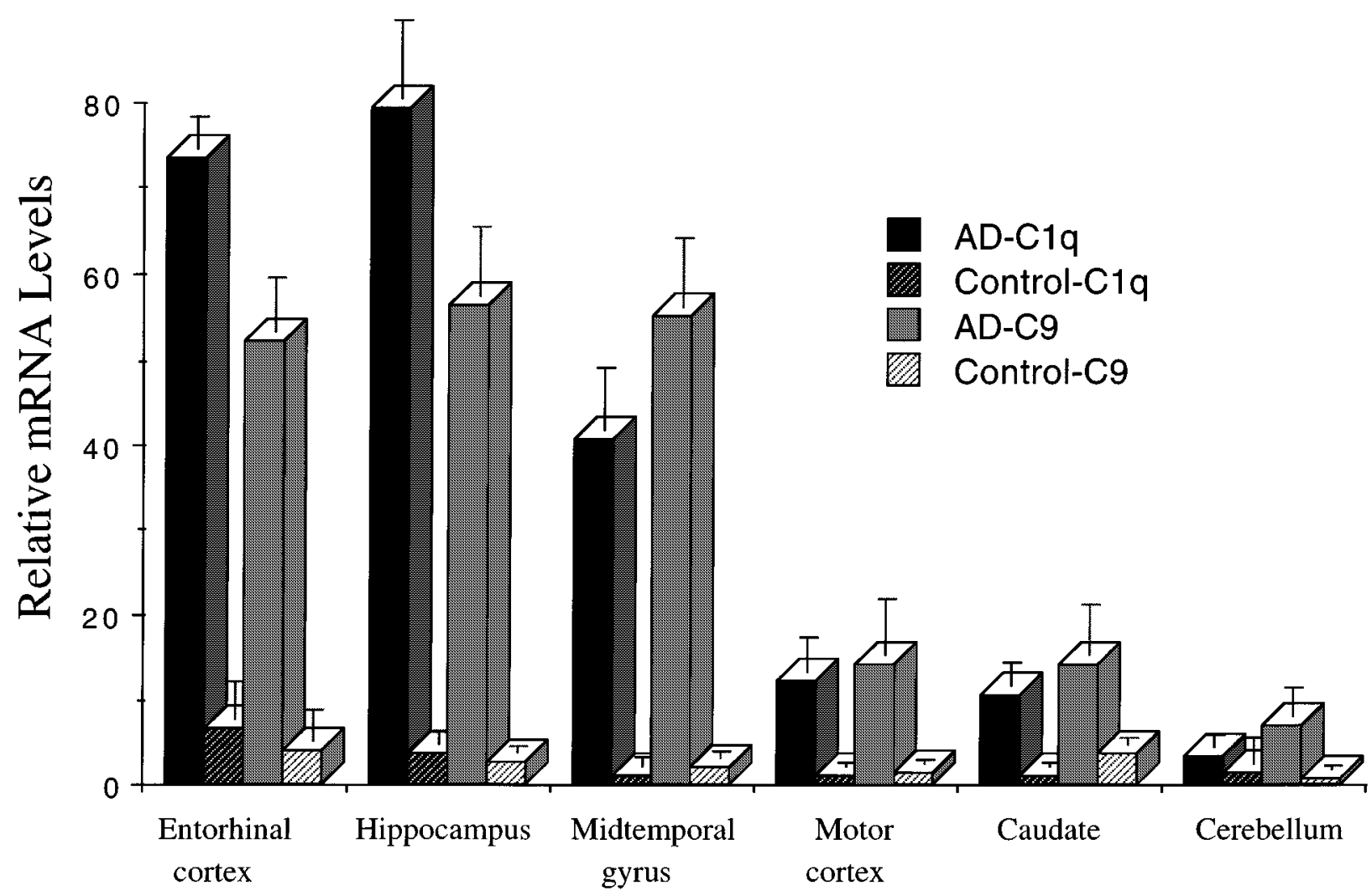

Figure 1 Relative levels for the mRNAs for C1 and C9, the first and last components of the complement cascade, in AD and control brain regions. Note the large increases in affected cortical areas in $\mathrm{AD}$, with much smaller (non-significant) changes in regions such as the caudate and cerebellum.

would seriously compromise the ability of the host to combat infection. One possibility that separates antibody activation of complement from that by the pentraxins or $\mathrm{A} \beta$ is $\mathrm{C} 1 \mathrm{q}$ binding. CRP, $\mathrm{AP}$, and $\mathrm{A} \beta$ all activate the complement cascade by binding to the collagen tail of C1q, whereas antibodies bind to the globular head (Jiang et al, 1992; Ying et al, 1993; Webster et al, 1997a). Thus, agents that block binding to the collagen tail of C1q might be effective therapeutic agents.

Examples of some inhibitors that block other steps in the complement cascade are the 13-residue cyclic peptide compstatin and the negatively charged sulfated glycosaminoglycan pentosan polysulfate (PPS). Compstatin binds to C3 and prevents its cleavage, thus inhibiting activation of both the classical and alternative complement pathways (Furlong et al, 2000). It has shown activity in a pig xenograft model (Fiane et al, 1999). PPS is an orally active, heparinlike compound that has been approved for the treatment of interstitial cystitis. It has been shown to prolong survival in a heterotopic rat cardiac transplant model (Schwartz et al, 1999) and to reduce myocardial infarct in a rabbit ischemia/reperfusion model (Tanhehco et al, 1999). Whether either would reach the brain in vivo is unknown; but these examples do demonstrate the possibility of designing synthetic complement inhibitors.

\section{Pentraxins}

Pentraxins are not generally considered to be inflammatory mediators. Yet they are ancient host-defense molecules that may function as primitive antibodylike compounds. When appropriately bound, they activate complement. They are believed to have evolved from an ancient gene more than 200,000,000 years ago. There are two molecules identified as pentraxins because of their unusual pentameric structure: CRP and AP.

CRP was originally identified as a host protein reacting with pneumococcal C polysaccharide, causing activation of the complement system. This gave an important clue to its function. It has since been learned that CRP, when appropriately bound, is a powerful activator of complement both in vivo and in viro (Jiang et al, 1992).

AP, the companion pentraxin, binds to all forms of amyloid. It also activates the complement system (Akiyama et al, 1991; Hicks et al, 1992).

The pentraxins meet the definition of acute phase reactants. Such reactants are defined as compounds whose serum levels increase or decrease by $25 \%$ or more following a general inflammatory reaction. In humans, CRP is a particularly sensitive acute phase reactant because its serum levels may rise as much as 1000 -fold following serious infection 
or injury. AP is the more sensitive reactant in rodents.

It was long believed that CRP and AP were products of the liver, but we have recently shown that both are produced by brain and are sharply up-regulated in areas damaged by $\mathrm{AD}$ (Yasojima et al, 2000). Neurons are the most prominent generators of both. CRP is associated with damaged fibers within AD senile plaques, whereas AP is associated with the extracellular amyloid deposits.

CRP is also produced in heart, where it is increased following myocardial injury, and in arteries where it is sharply up-regulated in atherosclerotic plaques (Yasojima et al, 2001). Serum CRP levels predict survival after heart attacks (Lagrand et al, 1999; Pietila et al, 1996) and strokes (Muir et al, 1999), and high normal CRP levels in apparently healthy individuals are associated with substantially increased odds of future adverse cardiovascular events (Ridker et al, 2000).

Taken together, these data suggest the following: (1) the pentraxins are secreted host-defense proteins produced by a variety of cells including neurons; (2) they may act like primitive antibodies by binding to foreign antigens or damaged host tissue, thus initiating appropriate complement attack; (3) they may extend their identification to host tissue inappropriate for attack, in which case an autotoxic reaction occurs; (4) agents that block binding of pentraxins to viable host tissue, or block their ability to activate complement, might be of therapeutic benefit in reducing autotoxic attack.

\section{Cytokines}

Cytokines are a heterogeneous group of small molecules that act in autocrine and paracrine fashion. They encompass several subfamilies, which include interleukins (ILs), interferons, tumor necrosis factors (TNFs), growth factors, colony-stimulating factors, and chemokines. They have in common participation in inflammatory reactions. They typically act in combination so that attributing a specific set of in vivo properties to any given cytokine is difficult.

Only a few of the cytokines have been extensively studied in AD. The most significant ones are IL- $1 \alpha$, IL- $1 \beta$, IL-6, and TNF- $\alpha$. The possibility that these inflammatory cytokines might play a role in inflammation in $\mathrm{AD}$ brain was initially suggested by the reports that they are all up-regulated in AD tissue and are prominently associated with AD lesions (Griffin et al, 1989; Wood et al, 1993; Dickson et al, 1993; Cacebelos et al, 1994). The inflammatory cytokines IL-1, IL-6, and TNF- $\alpha$ are products of both activated microglia and activated astrocytes and powerfully stimulate their activity. Localization of these cytokines to such activated cells has been demon- strated in AD by immunohistochemistry (Lieberman et al, 1989; Sharif et al, 1993; Yamabe et al, 1994; Walker et al, 1995).

Several reports have since appeared indicating that the risk of AD is substantially influenced by several polymorphisms in the noncoding regions of these inflammatory genes (Kamboh et al, 1997; Blacker et al, 1998; Papassotiropoulos et al, 1999; Collins et al, 2000; Du et al, 2000; Grimaldi et al, 2000; Licastro et al, 2000a, 2000b; Nicoll et al, 2000; Rebeck, 2000; McCusker, 2001). The polymorphisms are in promoter and untranslated regions. Those alleles, which favor increased expression of the inflammatory mediators, are more frequent in AD than in controls. The polymorphisms are fairly common ones in the general population, so there is a strong likelihood that any given individual will inherit one or more of the high-risk alleles. The odds ratio for a single one of these polymorphisms is much lower than for polymorphisms in apolipoprotein $\mathrm{E}$ (apoE), where different forms of the protein are expressed. Inheritance of the apoE4 form substantially increases the risk of AD (Strittmatter et al, 1993). However, McCusker (2001) has shown that carrying the high-risk allele of TNF- $\alpha$ substantially increases the risk of AD in carriers of the apoE4 allele. And it has also already been reported that the odds ratio is greatly increased if an individual carries two of the high-risk alleles. For example, Nicoll et al (2000) found that simultaneous inheritance of the high-risk alleles for IL$1 \alpha-889$ and IL- $\beta+3953$ increased the odds ratio for developing $\mathrm{AD}$ to 10.8; that is, the prevalence of $\mathrm{AD}$ in persons carrying these isoforms is 10.8 times as great as in persons of the same age carrying neither of these isoforms. The overall chances of an individual developing $\mathrm{AD}$ might be profoundly affected by a "susceptibility profile" reflecting the combined influence of inheriting multiple high-risk alleles (McGeer and McGeer, 2001). Identification of such profiles might in the future lead to strategies for therapeutic intervention in the very early stages of the disorder.

Each of these polymorphisms has also been linked to at least one peripheral inflammatory condition. The implication is that an inflammatory stimulus is more likely to cause autotoxic damage at vulnerable sites in numerous disorders in individuals carrying genetic polymorphisms that enhance inflammatory mediator expression.

The importance of $\mathrm{TNF}-\alpha$ as an inflammatory stimulus is emphasized by the widespread use of TNF- $\alpha$ antibodies or receptor blockers, such as infliximab and etanercept, in the treatment of rheumatoid arthritis. Both types of TNF- $\alpha$ inhibition induce a rapid improvement in multiple clinical measures of disease activity and patient functional status, as well as a beneficial effect on progression of joint damage as measured radiographically (Alldred, 2001; Hamilton and Clair, 2000). 


\section{Chemokines (Asensio and Campbell, 1999; Zlotnik and Yoshie, 2000)}

Chemokines are a large subfamily of cytokines of relatively low molecular weight (8 to $14 \mathrm{kDa}$ ). So far, over 50 members have been discovered. Their functions include chemotaxis and modulation of the activity of other classes of cytokines. Chemokines can be classified according to the number and spacing of amino terminal cysteines. On this basis, there are four distinct subfamilies: alpha (CXC), beta (CC), gamma (C), and delta (CX3C), where $\mathrm{C}$ stands for cysteine and $\mathrm{X}$ stands for a separating amino acid residue.

CC chemokines are primarily chemotactic for monocyte/macrophages; CXCs for neutrophils and endothelial cells; C (lymphotactin) for T cells and natural killer (NK) cells; and CX3C (fractalkine, neurotactin) for microglia.

Many of the chemokines were independently discovered by different groups. As a result, the same molecule may be referred to in the literature under several different names. Only some of the synonyms are mentioned in this review.

There are also multiple chemokine receptors to mediate their activities. They are G-protein linked. Again, there are subfamilies, which roughly correspond to their chemokine ligands. So far, there are five CXC receptors (CXC-R1 to CXC-R5), nine CC receptors (CC-R1 to CC-R9), plus single receptors for lymphotactin (XC-R1) and fractalkine (CX3C-R1). However, it should be noted that chemokines are not highly specific for given chemokine receptors and that much cross-reactivity occurs.

Because chemokines were originally identified as chemotactic agents for leukocytes, it is understandable that investigation of their role in the CNS has concentrated on infectious diseases and conditions where leukocyte infiltration is prominent, such as multiple sclerosis, experimental allergic encepholomyelitis (EAE), and stroke (reviewed in Bacon and Harrison, 2000). However, they may have a role to play in a much broader spectrum of neurodegenerative disorders where activated microglia affect the pathology. Of particular importance, therefore, are those chemokines and receptors that have a significant influence on microglial activity. Microglia have been shown to express the receptors CC-R3 and CC-R5 and this expression is up-regulated when microglia are activated. Abeta has been shown to stimulate the production of the chemokines IL-8 (CXCL8), monocyte chemotactic protein-1 (MCP-1, CCL2), macrophage inflammatory protein- $1 \alpha$ (MIP-1 $\alpha$, CCL3), and MIP-1 $\beta$ (CCL4) from human monocytes and IL-8, MCP-1, and MIP-1 $\alpha$ from autopsied human microglia (Neuroinflammation Working Group, 2000). MIP-1 $\beta$ has been identified on a subpopulation of human astrocytes.

Up-regulation of CXC-R2 has been observed on dystrophic neurites in AD (Hesselgesser and Horuk,
1999), and CC-R3 and CC-R5 have been identified on some reactive microglia in senile plaques (Xia et al, 1998).

Fractalkine and its receptor CX3CR-1 may prove to be a particularly interesting combination. Fractalkine is produced by neurons and exists in a bound as well as a free state. When neurons are exposed to excitotoxic damage, fractalkine is cleaved prior to neuronal death (Chapman et al, 2000). An antibody to fractalkine markedly reduced the neurotoxic effects of microglia stimulated by lipopolysaccharide (Zujovic et al, 2000). These data suggest that fractalkine might be a neuroprotective agent in vivo, being cleaved under neuronal stress and acting to reduce microglial activation. Agonists of CX3CR1 might therefore be effective anti-inflammatory agents.

Clearly research into defining the precise roles of chemokines in neuroinflammation in general, and $\mathrm{AD}$ in particular, is a priority for the future.

\section{Prostaglandins}

Prostaglandins are so named because they were originally discovered in the prostate gland. They are a group of fatty acids derived from the precursor arachidonic acid, with cyclooxygenase (COX) catalyzing the rate-controlling initial step of synthesis. Arachidonic acid itself is a product of phospholipase action on lipid membranes. Generally speaking, prostaglandins modulate the action of hormones, but they are now known to have a wide variety of functions. Among the most prominent of these is inflammatory mediation. This function has been the focus of intense therapeutic attention. Vane's group (Ferreira et al, 1973) opened up the field with his classic discovery that the antiinflammatory action of aspirin was due to its ability to inhibit prostaglandin synthesis. This formed the basis for developing a variety of COX-inhibiting agents, which are collectively described as nonsteroidal antiinflammatory drugs (NSAIDs). The failure of some of these agents to inhibit kidney prostaglandin synthesis led to the discovery of a second COX enzyme. The classical enzyme, located on chromosome 9, became known as cyclooxygenase-1 (COX-1) and the second enzyme, located on chromosome 1 , as cyclooxygenase-2 (COX-2). Although there is high homology between the two enzymes, the catalytic pockets differ so that inhibitors highly selective for each enzyme exist, whereas many of the traditional NSAIDs inhibit both forms. COX-1 is a relatively stable enzyme, whereas COX-2 is highly inducible, suggesting that at least some divergence in roles exists (McGeer, 2000). This is reinforced by significant differences in regional distribution. For example, COX-2 is highly expressed in kidney, whereas COX-1 is highly expressed in the gut (Yasojima et al, 1999c). 
As far as AD is concerned, both COX-1 and COX-2 mRNAs, as well as their protein products, are upregulated in affected areas of brain (Yasojima et al, 1999c). This is presumed to be largely due to inflammatory up-regulation of microglia, at least for COX-1. But, in contrast to COX-1, COX-2 is most abundantly expressed in pyramidal neurons, so the COX-2 response may be largely neuronal (Yasojima et al, 1999c). It has been suggested that COX-2 plays a vital role in synaptic plasticity (Kaufmann et al, 1996), which might be impaired by COX-2 inhibition. Studies of COX-2 inhibitors in various models of CNS damage have yielded conflicting results. Some indicate that COX-2 inhibition is harmful to the survival of neurons (Baik et al, 1999), whereas others suggest it may be neuroprotective (Nakayama et al, 1998). In either event, COX-2 is more intimately connected with pyramidal neurons than COX-1, and this should have implications as far as the use of these agents to reduce neuroinflammation in $\mathrm{AD}$ is concerned.

NSAIDs are the most widely used of all classes of drugs. As will be discussed later, they may have an important role to play in the treatment of $\mathrm{AD}$.

\section{Other inflammatory mediators}

The list of known inflammatory mediators that are up-regulated in AD is continuously expanding (for reviews listing partial tables, see McGeer and McGeer, 1995, 2001; Neuroinflammation Working Group, 2000). Many of the mediators are associated with reactive microglia. Such microglia surround the insoluble extracellular deposits of senile plaques and ghost tangles. But additional mediators are clearly associated with the activated astrocytes, which wall off the lesioned areas. Still others are associated with neurons, initially the producers of the extracellular material, but terminally the victims of autotoxic attack.

In addition to the classes of compounds previously discussed, there are proteases, particularly the metallomatrix proteinases; components of the coagulation pathways, that is, the thrombin and plastin systems; proteoglycans; cathepsins and cystatins; intercellular adhesion molecules; and such astrocytic products as $\mathrm{S}-100 \mathrm{~b}$ and $\alpha 1$-antichymotrypsin. Thus, the spectrum of molecules involved in AD neuroinflammation is very broad. Any of them might have a sufficiently powerful influence on the overall inflammatory state that blockade of their action might have therapeutic benefit. Insufficient knowledge is available to make accurate predictions regarding any of these relatively nonspecific inflammatory mediators, indicating that much further research is warranted.

In addition to proteins that mediate inflammation, free radicals are generated by the inflammatory process. The most abundant source of free radicals is activated microglia (Klegeris and McGeer, 2000). Microglia possess the NADPH oxidase complex, which, when assembled and fully activated, generates large numbers of free radicals on the external cell surface; these radicals are designed to destroy surrounding targets. A much smaller intracellular source of free radicals is leakage from the electron transport chain of mitochondria. In addition to oxygen free radicals from these sources, there is also glial generation of nitric oxide (Neuroinflammation Working Group, 2000). Nitric oxide can combine with oxygen free radicals to form the highly toxic product, peroxynitrite.

Footprints of oxygen free radical and peroxynitrite attack have been detected in postmortem $\mathrm{AD}$ tissue. These include the presence of proteins modified with advanced glycation end products, 4-hydroxynonenal, 8-hydroxyguanine, malondialdehyde, and nitrotyrosine (Neuroinflammation Working Group, 2000).

Activated microglia also possess the enzyme myeloperoxidase, which catalyzes a reaction between hydrogen peroxide, derived from oxygen free radicals, and chloride to generate the potent oxidizing agent hypochlorous acid (Reynolds et al, 1999). This system is most prominent in granulocytes where release of hypochlorous acid is one of their most potent attack weapons. Blocking myeloperoxidase may be one of the anti-inflammatory mechanisms of the antileprosy agent dapsone (Hope et al, 2000).

\section{Epidemiological and clinical evidence that inflammation plays a role in AD pathology}

If inflammation is playing a major role in converting $\mathrm{AD}$ from a relatively benign disorder into a malignant inflammatory condition, people taking antiinflammatory medications for other purposes might be inadvertently protecting themselves against the autotoxic effects of $\mathrm{AD}$. There are now more than 20 published epidemiological studies that show that people known to be taking anti-inflammatory agents or suffering from arthritis generally, or rheumatoid arthritis in particular, have their odds of developing AD considerably reduced (McGeer et al, 1996; Stewart et al, 1997; Veld et al, 2000; Broe et al, 2000). A revealing study was conducted by Stewart and his colleagues in Baltimore (1997). More than 2000 patients were enrolled in early middle age and followed for long periods of time to assess the factors contributing to later disease onset. The Stewart group therefore had records they could check regarding $A D$ and drugs. They found for those using NSAIDs for 2 years or less, the risk was reduced by about onethird. For those using NSAIDs for more than 2 years, the risk was reduced by $60 \%$. An even more thorough analysis was conducted by Veld et al (2001) on the data collected from the larger Rotterdam cohort of 6989 individuals. To determine actual NSAID use, pharmacy records were obtained on the 293 subjects who subsequently developed $\mathrm{AD}$. For those using NSAIDs for longer than 2 years, an $80 \%$ sparing of AD was found. This sparing was substantially greater 
than that found in the Baltimore study where actual pharmacy records were not obtained. The sparing was close to that originally reported by McGeer et al (1990) based on the prevalence of AD in rheumatoid arthritis patients who were presumed to be consuming therapeutic doses of anti-inflammatory agents on a long-term basis.

Can anti-inflammatory agents be used to treat $\mathrm{AD}$ ? Evidence from epidemiology suggests that they should be very effective. But for this possibility to be realized, appropriate agents must be selected. They should act on targets based on correlations between epidemiology and molecular pathology. The need for such correlations is illustrated by failures in clinical trails of prednisone, COX-2 inhibitors, and hydroxychloroquine (Aisen et al, 2000; Van Gool et al, 2001; Sainati et al, 2000). These agents were known to be effective in treating arthritic conditions but there was little or no evidence that they would be useful in neuroinflammation. MacKenzie (2000) provided data as to why steroids should fail based on assessment of the numbers of activated microglia in $\mathrm{AD}$ and control brain. Steroid consumption failed to reduce the number of reactive microglia detected postmortem. In contrast, there was a sharp reduction in reactive microglia in patients taking traditional NSAIDs. As far as selective COX-2 inhibitors are concerned, they have not been in use long enough for epidemiological data to accummulate, but immunohistochemical evidence that indicated they should fail was available. Unlike COX-1, COX-2 is highly expressed in normal pyramidal neurons, as well as in pyramidal neurons of AD cases (Yasojima et al, 1999c). Therefore, selective COX-2 inhibitors will primarily target pyramidal neurons rather than microglia. This, combined with evidence of exacerbation of neuronal death in some animal neurotoxicity models following COX-2 inhibition, suggests COX-2 inhibitors are an inappropriate choice. Hydroxyquinoline is not a general anti-inflammatory agent. Its mechanism of action in arthritis is uncertain. It is noteworthy, however, that its side effects involve the CNS. This includes both ototoxicity and retinal damage. Because there is no epidemiological or immunohistochemical evidence to support a role for hydroxychloroquine or other 4-aminoquinolines in neuroinflammation, the failure to be effective in $\mathrm{AD}$ might have been anticipated.

The situation with respect to traditional NSAIDs is quite different. The epidemiological evidence in favor of their protective effect is very strong. Moreover, one small, double-blind clinical trial of the COX-1 inhibitory NSAID indomethacin in established AD indicated arrest of progression (Rogers et al, 1993). Another NSAID trial, involving the mixed inhibitor diclofenac combined with misoprostyl, showed marginally less deterioration in drug compared with placebo patients, although the data fell short of statistical significance (Scharf et al, 1999). Moreover, in in vitro experiments, NSAIDs have been shown to reduce the neurotoxicity of activated microglia to cultured neuroblastoma cells (Klegeris et al, 1999). Clearly, properly designed clinical trials of COX-1-inhibiting NSAIDs that reach the brain should be a high priority for AD. Relatively high doses may be required due to the strong inflammatory reaction present in established AD. Gastrointestinal side effects are a problem with such agents, particularly at high doses. Although such side effects are significant, they are small in comparison with the inevitable progression and fatal outcome of $\mathrm{AD}$ with currently approved methods of treatment. It is possible that a new class of COX-1-inhibiting NSAIDs, based on introducing a nitrate ester moiety, may circumvent the side effect problem. These so-called NONSAIDs have greatly reduced gastrointestinal toxicity (Wallace et al, 1995).

Other types of anti-inflammatory agents may also be effective. An epidemiological study of leprosy patients in Japan showed that those continuously treated by dapsone had a significantly lower prevalence of dementia than those taken off dapsone for 5 years or more (McGeer et al, 1992). These data, combined with knowledge of the general antiinflammatory properties of dapsone, would suggest that dapsone might be effective in $\mathrm{AD}$, through a mechanism other than COX inhibition. Dapsone is a myeloperoxidase inhibitor and myeloperoxidase is an inflammatory mediator expressed by microglia. Again, epidemiological and neuropathological data can be correlated to suggest that dapsone might be effective in $\mathrm{AD}$, therefore warranting a clinical trial.

The spectrum of inlammatory mediators upregulated in $\mathrm{AD}$ suggests many routes for future therapeutic intervention. It may be that multiple drug administration, targeted at different inflammatory mechanisms, will prove far more effective than utilization of any single agent. In many cases, the data suggest targets not considered to be of importance in the immunological field. It may be that the lessons learned from $\mathrm{AD}$ research will have much broader applications in the general field of medicine.

\section{References}

Aisen PS, Davis KL, et al (2000). A randomized controlled trial of prednisone in Alzheimer's disease. Neurology 54: 588-593.

Akiyama H, Yamada T, et al (1991). Association of amyloid $\mathrm{P}$ component with complement proteins in neurologically diseased tissue. Brain Res 548: 349-352. 
Alldred A (2001). Etanercept in rheumatoid arthritis. Exp Opin Pharmacother 1: 1137-1148.

Asensio VC, Campbell IL (1999). Chemokines in the CNS: plurifunctional mediators in diverse states. Trends Neurosci 22: 504-512.

Bacon KB, Harrison JK (2000). Chemokines and their receptors in neurobiology: perspectives in physiology and homeostasis. J Neuroimmunol 104: 92-97.

Baik EJ, Kim EJ, et al (1999). Cyclooxygenase-2 selective inhibitors aggravate kainic acid induced seizure and neuronal cell death in the hippocampus. Brain Res 843: 118-129.

Blacker D, Wilcox MA, et al (1998). Alpha-2-macroglobulin is genetically associated with Alzheimer's disease. Nat Genet 19: 357-360.

Broe GA, Grayson DA, et al (2000). Anti-inflammatory drugs protect against Alzheimer's disease at low doses. Arch Neurol 57: 1586-1591.

Cacebelos R, Alvarez XA, et al (1994). Brain interleukin1 beta in Alzheimer's disease and vascular dementia. Methods Find Exp Clin Pharmacol 16: 141-145.

Chapman GA, Moores K, et al (2000). Fractalkine cleavage from neuronal membranes represents an acute event in the inflammatory response to excitotoxic brain damage. J Neurosci 20: 2-6.

Collin JS, Perry RT, et al (2000). Association of a haplotype for tumor necrosis factor in siblings with late-onset Alzheimer's disease: the NIMH Alzheimer's disease genetics initiative. Am J Med Genet 96: 823-830.

Dickson DW, Lee SC, et al (1993). Microglia and cytokines in neurological disease, with special reference to AIDS and Alzheimer's disease. Glia 7: 75-83.

$\mathrm{Du}$ Y, Dodel RC, et al (2000). Association of an interleukin 1 alpha polymorphism with Alzheimer's disease. Neurology 55: 480-483.

Eikelenboom P, Hack CE, et al (1989). Complement activation in amyloid plaques in Alzheimer's dementia. Virchows Arch Cell Pathol 56: 259-262.

Ferreira SH, Moncada S, Vane JR (1973). Prostaglandins and the mechanism of analgesia produced by aspirinlike drugs. Brit J Pharmacol 49: 86-97.

Fiane AE, Mollnes TE, et al (1999). Compstatin, a peptide inhibitor of C3, prolongs survival of ex vivo perfused pig xenografts. Xenotransplantation 6: 52-65.

Furlong ST, Dutta AS, et al (2000). C3 activation is inhibited by analogs of compstatin but not by serine protease inhibitors or peptidyl alpha-ketoheterocycles. Immunopharmacology 48: 199-212.

Griffin WST, Stanley LC, et al (1989). Brain interleukin 1 and S-100 immunoreactivity are elevated in Down syndrome and Alzheimer's disease. Proc Soc Natl Acad Sci USA 86: 7611-7615.

Grimaldi LM, Casadei VM, et al (2000). Association of early-onset Alzheimer's disease with an interleukin-1 $\alpha$ gene polymorphism. Ann Neurol 47: 361-365.

Hamilton K, Clair EW (2000). Tumour necrosis factor-alpha blockade: a new era for the effective management of rheumatoid arthritis. Exp Opin Pharmacother 1: 10411052.

Haymaker W, Adams RD (eds) (1982). Histology and histopathology of the nervous system. Charles $\mathrm{C}$ Thomas: Springfield, IL, pp 1150-1173.

Hesselgesser J, Horuk R (1999). Chemokine and chemokine receptor expression in the central nervous system. J Neurovirol 5: 13-26.
Hicks PS, Saunero-Nazia L, et al (1992). Serum amyloid P component binds to histones and activates the classical complement pathway. J Immunol 149: 3689-3694.

Hope HR, Remsen EE, et al (2000). Large-scale purification of myeloperoxidase from KL60 promyelocytic cells: characterization and comparison to human neutrophil myeloperoxidase. Protein Expr Purif 18: 269-276.

Ishii T, Haga S (1984). Immuno-electron-microscopic localization of complements in amyloid fibrils of senile plaques. Acta Neuropathol (Berlin) 63: 296-300.

Itagaki S, Akiyama $\mathrm{H}$, et al (1994). Ultrastructural localization of complement membrane attack complex (MAC)-like immunoreactivity in brains of patients with Alzheimer's disease. Brain Res 645: 78-84.

Jiang H, Robey F, et al (1992). Localization of sites through which C-reactive protein binds to and activates complement to residues 14-26 and 76-92 of the human C1q A chain. J Exp Med 175: 1373-1379.

Kamboh MI, Aston CE, et al (1997). Genetic effect of alpha1-antichymotrypsin on the risk of Alzheimer's disease. Genomics 40: 382-384.

Kaufmann WE, Worley PF, et al (1996). COX-2, a synaptically induced enzyme, is expressed by excitatory neurons at post-synaptic sites in rat cerebral cortex. Proc Natl Acad Sci USA 93: 2317-2321.

Klegeris A, McGeer PL (2000). Interaction of various intracellular signaling mechanisms involved in mononuclear phagocyte toxicity toward neuronal cells. J Leukoc Biol 67: 127-133.

Klegeris A, Walker DG, et al (1999). Toxicity of human THP-1 monocytic cells towards neuron-like cells is reduced by non-steroidal anti-inflammatory drugs (NSAIDs). Neuropharmacology 38: 1017-1025.

Lagrand WK, Visser CA, et al (1999). C-reactive protein as a cardiovascular risk factor. Circulation 100: 96102.

Lambris DH (1993). The chemistry, biology, and phylogeny of C3. In: Complement today. Cruise JM, LLewis RE Jr (eds). Karger: Basel, pp 16-45.

Licastro F, Pedrini S, et al (2000a). Polymorphisms of the IL-6 gene increase the risk for late onset Alzheimer's disease and affect IL-6 plasma levels. Neurobiol Aging 21(1S): S38.

Licastro F, Pedrini S, et al (2000b). Gene polymorphism affecting $\alpha 1$-antichymotrypsin and interleukin-1 plasma levels increases Alzheimer's disease risk. Ann Neurol 48: 388-391.

Lieberman AP, Pitha PM, et al (1989). Production of tumor necrosis factor and other cytokines by astrocytes stimulated with lipopolysaccharide or a neurotropic virus. Proc Natl Acad Sci USA 86: 6348-6352.

MacKenzie IRA (2000). Anti-inflammatory drugs and Alzheimer-type pathology in aging. Neurology 54: 732734.

McCusker SM (2001). Association between polymorphism in regulatory region of gene encoding tumour necrosis factor A and risk of Alzheimer's disease and vascular dementia: a case-control study. Lancet 357: 436439.

McGeer EG, Yasojima K, et al (2001). Inflammation in the pathogenesis of Parkinson's disease. BC Med J 43: 138141.

McGeer PL (2000). Cyclooxygenase-2 inhibitors: rationale and therapeutic potential for Alzheimer's disease. Drugs Aging 17: 1-11. 
McGeer PL, Akiyama H, et al (1989). Immune system response in Alzheimer's disease. Can J Neurol Sci 16: 516527.

McGeer PL, Harada N, et al (1992). Prevalence of dementia amongst elderly Japanese with leprosy: apparent effect of chronic drug therapy. Dementia 3: 146-149.

McGeer PL, McGeer EG (1995). The inflammatory response system of brain: implications for therapy of Alzheimer's and other neurodegenerative diseases. Brain Res Rev 21: 195-218.

McGeer PL, McGeer EG (2000). Autotoxicity and Alzheimer's disease. Arch Neurol 57: 789-790.

McGeer PL, McGeer EG (2001). Polymorphisms in inflammatory genes enhance the risk of Alzheimer's disease. Arch Neurol 58: 1790-1792.

McGeer PL, Rogers J, et al (1990). Anti-inflammatory drugs and Alzheimer's disease? Lancet 335, 1037.

McGeer PL, Schulzer M, et al (1996). Arthritis and antiinflammatory agents as possible protective factors for Alzheimer's disease: a review of 17 epidemiological studies. Neurology 47: 425-432.

Muir KW, Weir CJ, et al (1999). C-reactive protein and outcome after ischemic stroke. Stroke 30: 981-985.

Nakayama M, Uchimura K, et al (1998). Cyclooxygenase-2 inhibition prevents delayed death of CA1 hippocampal neurons following global ischemia. Proc Natl Acad Sci USA 95: 10954-10959.

Neuroinflammation Working Group (2000). Inflammation and Alzheimer's disease. Neurobiol Aging 21: 383-421.

Nicoll JAR, Mrak RE, et al (2000). Association of interleukin-1 gene polymorphisms with Alzheimer's disease. Ann Neurol 47: 365-368.

Papassotiropoulos A, Bagli M, et al (1999). A genetic variation of the inflammatory cytokine interleukin-6 delays the initial onset and reduces the risk for sporadic Alzheimer's disease. Ann Neurol 45: 666-668.

Pietila KO, Harmoinen AP, et al (1996). Serum C-reactive protein concentration in acute myocardial infarction and its relationship to mortality during 24 months of follow-up in patients under thrombolytic treatment. Eur Heart J 17: 1345-1349.

Rebeck GW (2000). Confirmation of the genetic association of interleukin-1A with early onset sporadic Alzheimer's disease. Neurosci Lett 293: 75-77.

Reynolds WF, Rhees J, et al (1999). Myeloperoxidase polymorphism is associated with gender specific risk for Alzheimer's disease. Exp Neurol 155: 31-41.

Ridker PM, Hennekens CH, et al (2000). C-reactive protein and other markers of inflammation in the prediction of cardiovascular disease in women. $N$ Engl J Med 342: 836-843.

Rogers J, Cooper NR, et al (1992). Complement activation by $\beta$-amyloid in Alzheimer's disease. Proc Natl Acad Sci USA 89: 10016-10020.

Rogers J, Kirby LC, et al (1993). Clinical trial of indomethacin in Alzheimer's disease. Neurology 43: 1609-1611.

Sainati SM, Ingram DM, et al (2000). Results of a doubleblind, placebo-controlled study of celecoxib for the progression of Alzheimer's disease. In: Proceedings of 6th International Stockholm-Springfield Symposium of Advances in Alzheimer's Therapy. p 180.

Scharf S, Mander A, et al (1999). A double-blind, placebocontrolled trial of diclofenac misoprostol in Alzheimer's disease. Neurology 53: 197-201.
Schwartz CF, Kilgore KS, et al (1999). Increased rat cardiac allograft survival by the glycosaminoglycan pentosan polysulfate. J Surg Res 86: 24-28.

Sharif SF, Hariri RJ, et al (1993). Human astrocyte production of tumour necrosis factor-alpha, interleukin-1 beta, and interleukin-6 following exposure to lipopolysaccharide endotoxin. Neurol Res 15: 109-112.

Stewart WF, Kawas C, et al (1997). Risk of Alzheimer's disease and duration of NSAID use. Neurology 48: 626-632.

Strittmatter WJ, Saunders A, et al (1993). Apolipoprotein $\mathrm{E}$ : high avidity binding to $\beta$-amyloid and increased frequency of type 4 allele in late-onset familial Alzheimer's disease. Proc Natl Acad Sci USA 90: 1977-1981.

Tanhehco EJ, Kilgore KS, et al (1999). Reduction of myocardial infarct size after ischemia and reperfusion by the glycosaminoglycan pentosan polysulfate. J Cardiovasc Pharmacol 34: 153-161.

Van Gool WA, Weinstein HC, et al (2001). Effect of hydroxychloroquine on progression of dementia in early Alzheimer's disease: an 18-month randomized, doubleblind, placebo-controlled study. Lancet 358: 455-460.

Veld BAI, Ruitenberg A, et al (2000). Duration of non-steroidal antiinflammatory drug use and risk of Alzheimer's disease. The Rotterdam study. Neurobiol Aging 21(1S): S204.

Veld BAI, Ruitenberg A, et al (2001). Nonsteroidal antiinflammatory drugs and the risk of Alzheimer's disease. New Engl J Med 345: 1515-1521.

Walker DG, Kim SU, et al (1995). Complement and cytokine gene expression in cultured microglia derived from postmortem human brains. J Neurosci Res 40: 478-493.

Wallace JL, Pittman QJ, et al (1995). Nitric oxide-releasing NSAIDs: a novel class of GI-sparing anti-inflammatory drugs. Agents Actions 46(Suppl): 121-129.

Webster S, Bonnell B, et al (1997a). Charge-based binding of complement component C1q to the Alzheimer's amyloid beta-peptide. Am J Pathol 150: 1531-1536.

Webster S, Lue LF, et al (1997b). Molecular and cellular characterization of the membrane attack complex, C5b-9, in Alzheimer's disease. Neurobiol Aging 18: 415421.

Wood JA, Wood PL, et al (1993). Cytokine indices in Alzheimer's temporal cortex: no change in mature IL beta or IL-1RA but increases in the associated acute phase proteins IL-6, alpha 2-macroglobulin and C-reactive protein. Brain Res 629: 245-252.

Xia MQ, Qin SX, et al (1998). Immunohistochemical study of the beta-chemokine receptors CCR3 and CCR5 and their ligands in normal and Alzheimer's disease brains. Am J Pathol 153: 31-37.

Yamabe T, Dhir G, et al (1994). Cytokine-gene expression in measles-infected adult human glial cells. J Neuroimmunol 49: 171-179.

Yasojima K, McGeer EG, et al (1999a). Complement regulators C1 inhibitor and CD59 do not significantly inhibit complement activation in Alzheimer's disease. Brain Res 833: 297-301.

Yasojima K, Schwab C, et al (1998). Human heart generates complement proteins that are upregulated and activated after myocardial infarction. Circ Res 83: 860869.

Yasojima K, Schwab C, et al (1999b). Upregulated production and activation of the complement system in Alzheimer's disease brain. Am J Pathol 154: 927936. 
Yasojima K, Schwab C, et al (1999c). Distribution of cyclooxygenase-1 and cyclooxygenase-2 mRNAs and proteins in human brain and peripheral organs. Brain Res 830: 226-236.

Yasojima K, Schwab C, et al (2000). Human neurons generate C-reactive protein and amyloid P: upregulation in Alzheimer's disease. Brain Res 887: 8089.

Yasojima K, Schwab C, et al (2001). Generation of C-reactive protein and complement components in atherosclerotic plaques. Am J Pathol 158: 1039-1051.
Ying SC, Gewurz AT, et al (1993). Human serum amyloid $\mathrm{P}$ component oligomers bind and activate the classical complement pathway via residues $14-26$ and 76-92 of the A chain collagen-like region of C1q. J Immunol 150: 169-176.

Zlotnik A, Yoshie O (2000). Chemokines: a new classification system and their role in immunity. Immunity 12: 121-127.

Zujovic V, Benavides J, et al (2000). Fractalkine modulates TNF-alpha secretion and neurotoxicity induced by microglial activator. Glia 29: 305-315. 